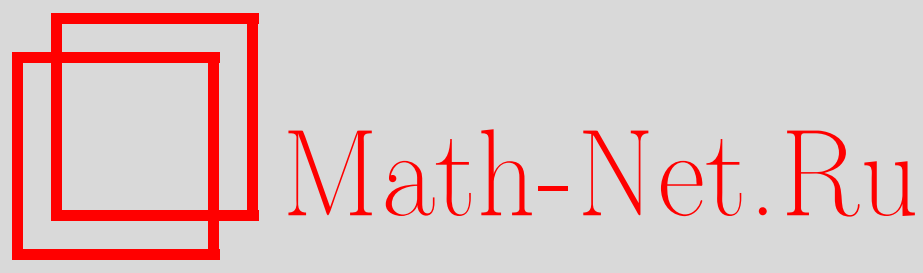

В. Е. Захаров, А. В. Одесский, М. Цистернино, М. Онорато, Пятиволновая классическая матрица рассеяния и интегрируемые уравнения, ТМФ, 2014, том 180, номер 1, 10-16

DOI: https://doi.org/10.4213/tmf8639

Использование Общероссийского математического портала Math-Net.Ru подразумевает, что вы прочитали и согласны с пользовательским соглашением http://www.mathnet.ru/rus/agreement

Параметры загрузки:

IP : 35.173 .219 .12

26 апреля 2023 г., 13:16:15

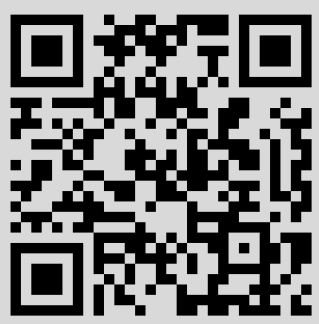




\title{
ПЯТИВОЛНОВАЯ КЛАССИЧЕСКАЯ МАТРИЦА РАССЕЯНИЯ И ИНТЕГРИРУЕМЫЕ УРАВНЕНИЯ
}

\begin{abstract}
Исследуется пятиволновая классическая матрица рассеяния для нелинейных гамильтоновых уравнений с дисперсией, характеризуемых нелинейностью вида $u \partial u / \partial x$, с целью найти наиболее общий вид нетривиального дисперсионного соотношения $\omega(k)$, для которого пятиволновая матрица рассеяния для взаимодействия тождественно обращается в нуль на резонансном многообразии. Как можно было ожидать, в размерности 1 матрица рассеяния тождественно обращается в нуль в случаях уравнения Кортевега-де Фриза, уравнения Бенджамина-Оно и уравнения промежуточных длинных волн. В размерности 2 найдено новое уравнение, удовлетворяющее указанным условиям.
\end{abstract}

Ключевые слова: интегрируемость, уравнение промежуточных длинных волн, уравнение Кортевега-де Фриза, уравнение Бенджамина-Оно, матрица рассеяния.

DOI: $10.4213 / \operatorname{tmf} 8639$

За последние 40 лет в области нелинейных уравнений был достигнут значительный прогресс. Развитие новых математических методов привело к выделению класса интегрируемых нелинейных уравнений в частных производных. Эти нелинейные интегрируемые уравнения играют важнейшую роль при исследовании физических систем. Применяя асимптотические методы, использующие малые параметры, характеризующие интересующий нас физический режим, можно свести к интегрируемым уравнениям весьма широкий класс нелинейных эволюционных уравнений (см. [1]). Различные методы были развиты для установления свойств этих уравнений [2]. В этом отношении данная работа базируется на теореме Захарова-Шульмана (см. [3]-[5]), которая связана с анализом Пуанкаре интегрируемости динамических систем. Эта теорема основана на теории возмущений и на введении так

${ }^{*}$ University of Arizona, Tucson, USA

${ }^{\dagger}$ Физический институт им. П. Н. Лебедева РАН, Москва, Россия.

E-mail: zakharov@itp.ac.ru

${ }^{\ddagger}$ Новосибирский государственный университет, Новосибирск, Россия

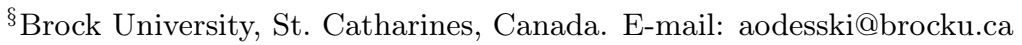

`Dipartimento di Fisica, Università di Torino, Torino, Italy

"INFN, Sezione di Torino, Torino, Italy 
называемой классической матрищы рассеяния, которая в случае классической гамильтоновой системы связывает два асимптотических состояния $(t \rightarrow \pm \infty)$. Если говорить нестрого, теорема утверждает, что существование одного дополнительного интеграла движения приводит к тому, что для всякого резонансного процесса матрица рассеяния обращается к нуль; теорема также утверждает, что существует бесконечный набор инвариантов. Этого еще, конечно, недостаточно для интегрируемости (для которой еще надо доказать полноту множества инвариантов). Одним из следствий теоремы оказывается утверждение о том, что для неинтегрируемой системы матрица рассеяния не равна тождественно нулю. Этот результат был недавно применен, например, при доказательстве неинтегрируемости компактного одномерного уравнения Захарова [6].

В настоящей работе обсуждается интегрируемость некоторых волновых уравнений гидродинамического типа. В частности, хорошо известно, что уравнение Кортевега-де Фриза (КдФ), уравнение Бенджамина-Оно (БО) и уравнение промежуточных длинных волн (ПДВ) доставляют примеры интегрируемых систем, для которых нелинейный оператор имеет один и тот же вид, а линейные дисперсионные члены оказываются различными. Применяя теорему Захарова-Шульмана, можно исследовать вопрос о том, существуют ли другие интегрируемые гамильтоновы волновые уравнения, характеризуемые тем же самым нелинейным оператором. Похожая задача исследовалась в работах [7], [8].

Рассмотрим следующее волновое уравнение общего вида:

$$
\frac{\partial u}{\partial t}+u \frac{\partial u}{\partial x}+\omega_{k} u=0
$$

где $u$ - вещественная функция переменной $x$, а $\omega_{k}$ - оператор свертки, ответственный за волновую дисперсию. Будем считать $\omega_{k}$ нечетной функцией от $k$, т. е. $\omega(k)=$ $-\omega(-k)$, и $\omega(0)=0$. Уравнение $(1)$ в фурье-пространстве можно записать в виде

$$
i \frac{\partial u_{1}}{\partial t}=\omega_{1} u_{1}+k_{0} \int_{-\infty}^{\infty} u_{2} u_{3} \delta_{1-2-3} d k_{23}
$$

где $u_{i}=u\left(k_{i}\right), d k_{23}=d k_{2} d k_{3}, \delta_{1-2-3}=\delta\left(k_{1}-k_{2}-k_{3}\right)$. Введем теперь нормальную переменную $a(k)$, связанную с $u(k)$ :

$$
u_{k}=\sqrt{k}\left(a_{k} \theta_{k}+a_{-k}^{*} \theta_{-k}\right),
$$

где $\theta_{k}=\theta(k)$ - функция ступеньки Хевисайда. В терминах этой новой переменной уравнение приобретает следующий гамильтонов вид:

$$
H=H_{0}+H_{\text {int }}=\int_{0}^{\infty} \omega_{1}\left|a_{1}\right|^{2} d k_{1}+\int_{0}^{\infty} V_{1,2,3}\left(a_{1} a_{2}^{*} a_{3}^{*}+a_{1}^{*} a_{2} a_{3}\right) \delta_{1-2-3} d k_{123},
$$

где $V_{1,2,3}=\sqrt{k_{1} k_{2} k_{3}} \theta_{1} \theta_{2} \theta_{3}$.

Эволюционное уравнение записывается в виде

$$
i \frac{\partial a_{k}}{\partial t}=\frac{\delta H}{\delta a_{k}^{*}} .
$$

В случае произвольной гамильтоновой системы вида (4) гамильтониан (3) можно заменить на вспомогательный гамильтониан $H_{\varepsilon}=H_{0}+H_{\mathrm{int}} e^{-\varepsilon|t|}$ с $\varepsilon>0$ и перейти 
к пределу $t \rightarrow \pm \infty$ в (4). При произвольных начальных условиях $\left.a(k, t)\right|_{t=0}$ асимптотические поля $\left.a(k, t)\right|_{t \rightarrow \pm \infty}$ задаются формулами $c_{\varepsilon}(k)^{ \pm} e^{-i \omega(k) t}$. Эти асимптотические предельные выражения не независимы и связаны соотношениями $c_{\varepsilon}(k)^{+}=$ $S_{\varepsilon}\left[c_{\varepsilon}(k)^{-}\right]$, в которых $S_{\varepsilon}-$ нелинейный оператор, задаваемый рядом, сходящимся при достаточно больших $\varepsilon$. Определим классическую матричу рассеяния (подробнее см. [3]) с помощью предельного перехода $\varepsilon \rightarrow 0$, а именно

$$
S=\lim _{\varepsilon \rightarrow 0} \widehat{S}_{\varepsilon}, \quad c_{\varepsilon}^{ \pm}(k) \rightarrow c^{ \pm}(k) .
$$

После взятия предела ряд может оказаться расходящимся; в этом случае его следует понимать как формальный ряд, коэффициенты которого $c^{+}(k)$ и $c^{-}(k)$ связаны соотношениями

$$
\begin{aligned}
c^{+}\left(k_{1}\right)=S c^{-}\left(k_{1}\right)= & c^{-}\left(k_{1}\right)+\sum_{n=3}^{\infty} \sum_{s_{1}, \ldots, s_{n}} \int_{0}^{\infty} S_{1,2, \ldots, n} \times \\
& \times \delta\left(s_{1} k_{1}+s_{2} k_{2}+\cdots+s_{n} k_{n}\right) c_{1}^{-s_{2}} \ldots c_{n}^{-s_{n}} d k_{2 \ldots n} .
\end{aligned}
$$

Величины $s_{i}$ принимают значения +1 или $-1, c^{ \pm s}$ равны $c^{ \pm}$при $s=1$ и равны $\left(c^{ \pm}\right)^{*}$ при $s=-1$. Классическая матрица рассеяния принимает следующий вид:

$$
S_{1,2, \ldots, n}=i T_{1,2, \ldots, n} \delta\left(s_{1} \omega_{1}+s_{2} \omega_{2}+\cdots+s_{n} \omega_{n}\right),
$$

где $T_{1,2, \ldots, n}$ - амплитуда рассеяния, заданная на поверхности

$$
\begin{aligned}
s_{1} \omega_{1}+s_{2} \omega_{2}+\cdots+s_{n} \omega_{n} & =0, \\
s_{1} k_{1}+s_{2} k_{2}+\cdots+s_{n} k_{n} & =0 .
\end{aligned}
$$

В зависимости от величины $s_{i}$ приведенные выше условия разбиваются в набор условий, задающих резонансное многообразие. Захаров и Шульман [3] доказали важную теорему, утверждающую, что в случае, когда система (4) обладает дополнительным (помимо энергии, импульса и массы) интегралом движения вида

$$
I=\int f_{k}\left|a_{k}\right|^{2} d k+\cdots
$$

где многоточием обозначены члены высших порядков по $a_{k}$, и при этом дисперсионное соотношение оказывается невырожденным, для каждого процесса рассеяния, отличаемого выбором $s_{i}$ в (8), амплитуда рассеяния $T_{0,1, \ldots, n}=0$ и в системе появляется бесконечное количество интегралов движения, находящихся в инволюции. Эта теорема не позволяет непосредственно сделать заключение об интегрируемости данного уравнения; тем не менее для данной гамильтоновой системы вида (4) можно установить, что наличие нетривиального рассеяния, т. е. выполнение условия $T_{1,2, \ldots, n} \neq 0$ на некотором резонансном многообразии, явно свидетельствует о неинтегрируемости системы.

В специальном случае уравнение (1) описывает волны, распространяющиеся в одном направлении, а потому в формулировке нормальных переменных интегралы в гамильтониане (3) определены при $k \geqslant 0$. Наша задача состоит в том, чтобы найти $\omega(k)$ специального вида, при котором амплитуда рассеяния обращается в нуль 
на резонансном многообразии. Для волновых процессов с участием трех и четырех волн получаются только тривиальные решения, так что первым нетривиальным случаем оказывается пятиволновой процесс. В этом случае резонансное многообразие задается следующими уравнениями:

$$
\begin{gathered}
k_{4}+k_{5}=k_{1}+k_{2}+k_{4}, \\
\omega\left(k_{4}\right)+\omega\left(k_{5}\right)=\omega\left(k_{1}\right)+\omega\left(k_{2}\right)+\omega\left(k_{3}\right) .
\end{gathered}
$$

Матрица рассеяния задается громоздким выражением, содержащим 80 слагаемых (см. работу [9], в которой была разработана диаграммная техника построения этих членов). Предполагается, что все положительные волновые числа упорядочены следующим образом:

$$
k_{2}>k_{4}>k_{5}>k_{3}>k_{1} .
$$

При таком предположении матрица рассеяния пятиволновой амплитуды значительно упрощается и принимает вид

$$
\begin{aligned}
T_{123-4-5}= & F_{12}\left(F_{45}+G_{53}+G_{43}\right)+F_{13}\left(F_{45}+G_{25}+G_{24}\right)+G_{51}\left(F_{23}+G_{43}+G_{24}\right)+ \\
& +G_{41}\left(F_{23}+G_{53}+G_{25}\right)+F_{45} F_{23}+G_{24} G_{53}+G_{25} G_{43}
\end{aligned}
$$

где

$$
F_{i j}=\frac{k_{i}+k_{j}}{\omega\left(k_{i}+k_{j}\right)-\omega\left(k_{i}\right)-\omega\left(k_{j}\right)}, \quad G_{i j}=\frac{k_{i}-k_{j}}{\omega\left(k_{i}-k_{j}\right)-\omega\left(k_{i}\right)+\omega\left(k_{j}\right)}
$$

с $i \neq j=1, \ldots, 5$. Необходимым условием интегрируемости оказывается сокращение пятиволновой амплитуды на резонансном многообразии (9).

Ниже показано, что для уравнений КдФ, БО и ПДВ это свойство выполняется.

Уравнение КдФ. Пусть $\omega(k)=-k^{3}$, тогда

$$
F_{i j}=\frac{1}{3 k_{i} k_{j}}, \quad G_{i j}=-\frac{1}{3 k_{i} k_{j}} .
$$

В результате простого вычисления для матрицы рассеяния пятиволновой амплитуды получаем

$$
T_{123-4-5}=\frac{1}{9 k_{1} k_{2} k_{3} k_{4} k_{5}}\left(k_{4}+k_{5}-k_{1}-k_{2}-k_{3}\right)=0 .
$$

Заметим, что $T_{123-4-5}=0$ даже вне резонансного многообразия (необходимо лишь выполнение условия сохранения импульса), т. е. ограничение на частоты (9) не обязательно соблюдается.

Уравнение БО. Уравнение БО моделирует эволюцию длинных одномерных внутренних гравитационных волн в стратифицированной жидкости в слое бесконечной глубины. Для такого уравнения получим, что $\omega(k)=-|k| k$, и для положительных волновых чисел можно положить $\omega(k)=-k^{2}$. Тогда

$$
F_{i j}=\frac{k_{i}+k_{j}}{2 k_{i} k_{j}}, \quad G_{i j}=-\frac{1}{2 k_{j}}
$$


и можно показать, что пятиволновая амплитуда рассеяния задается выражением

$$
T_{123-4-5}=L_{12345}\left(k_{4}^{2}+k_{5}^{2}-k_{1}^{2}-k_{2}^{2}-k_{3}^{2}\right)+M_{12345}\left(k_{1}+k_{2}+k_{3}-k_{4}-k_{5}\right),
$$

где $L_{12345}$ и $M_{12345}$ - две положительные функции от волновых чисел. Тем самым сокращение матрицы рассеяния, очевидно, следует из уравнений (9).

Уравнение ПДВ. Уравнение ПДВ описывает длинные внутренние гравитационные волны в стратифицированной жидкости конечной глубины. Дисперсионное соотношение имеет вид

$$
\omega(k)=a k^{2} \operatorname{cth} b k-c k
$$

и сводится к уравнению КдФ в пределе $b \rightarrow 0, a=1$ и $c=0$ и к уравнению БО в пределе $b \rightarrow \infty, a=3 / b$ и $c=3 / b^{2}$. Чтобы проверить сокращение такого дисперсионного соотношения, заметим сначала, что выражение $T_{123-4-5}$ инвариантно относительно замены $\omega(k) \rightarrow \omega(\alpha k)+\beta k$, где $\alpha \neq 0$ и $\beta$ - произвольные константы. Более того, можно ввести дополнительную независимую переменную $p$ и положить

$$
\omega(k) \rightarrow \omega(k, p)=k^{2} \frac{1+e^{p}}{1-e^{p}} .
$$

Резонансная поверхность должна удовлетворять следующим уравнениям:

$$
\begin{gathered}
p_{4}+p_{5}=p_{1}+p_{2}+p_{3}, \quad k_{4}+k_{5}=k_{1}+k_{2}+k_{3}, \\
\omega_{k_{4}, p_{4}}+\omega_{k_{5}, p_{5}}=\omega_{k_{1}, p_{1}}+\omega_{k_{2}, p_{2}}+\omega_{k_{3}, p_{3}} .
\end{gathered}
$$

Теперь матрица рассеяния пятиволновой амплитуды зависит от десяти переменных, $T_{12345}=T\left(k_{1}, \ldots, k_{5}, p_{1}, \ldots, p_{5}\right)$, и сокращение этой матрицы на резонансном многообразии было успешно проверено с помощью символьных вычислений с применением программы Maple. Обратим теперь внимание на наиболее общий случай и рассмотрим

$$
T\left(k_{1}, \ldots, k_{5}\right)=0
$$

как функциональное уравнение на неизвестную функцию $\omega(k)$ с волновыми векторами $k_{1}, \ldots, k_{5}$, удовлетворяющими условию резонанса (9). Напомним, что в случае, когда $\omega(k)$ представляет собой решение функционального уравнения, функция

$$
\tilde{\omega}(k)=a \omega(b k)+c k
$$

также оказывается решением при произвольных $a, b \neq 0$ и $c$.

Сформулируем следующее

ПрЕДЛОЖЕНИЕ 1. Произвольное решение функционалъного уравнения, аналитическое в окрестности нуля и такое, что $\omega(0)=0$, равно одной из следующих функицй: $\omega_{1}(k)=k^{2}, \omega_{2}(k)=k^{3}$ или $\omega_{3}(k)=-k^{2}\left(e^{2 k}+1\right) /\left(e^{2 k}-1\right)=k^{2} \operatorname{cth} k$. Предполагая $a, b \neq 0$ комплексными числами, получим, что существует четвертое решение $\omega_{4}(k)=i k^{2}\left(e^{i 2 k}+1\right) /\left(e^{i 2 k}-1\right)=-k^{2} \operatorname{ctg} k$.

ДокАЗАТЕЛьство. Идея доказательства состоит в сведении функционального уравнения (19) к обыкновенному дифференциальному уравнению с последующим нахождением его решений. Положим $k_{2}=k_{4}+u, k_{5}=k_{3}+v$, тогда условие сохранения импульса в (9) приводит к условию $k_{1}=v-u$. Рассматривая теперь 
ограничения на частоты (условие сохранения энергии в (9)) и разлагая в ряд Тейлора вблизи $u=v=0$, получим

$$
v=\frac{\omega^{\prime}\left(k_{4}\right)}{\omega^{\prime}\left(k_{3}\right)} u+o(u) .
$$

Более того, разлагая функциональное уравнение (19) в окрестности $u=v=0$ и применяя уравнение (21), получим обыкновенное дифференциальное уравнение, которое в ведущем порядке содержит большое количество слагаемых. Без потери общности, в предположении, что $\omega(k)=a_{2} k^{2}+a_{3} k^{3}+\cdots$, в разложении обыкновенного дифференциального уравнения в окрестности $k_{3}=k_{4}=0$ получим условие $a_{2} a_{3}=0$, приводящее к следующим случаям.

СлУчАй 1 . Пусть $a_{2} \neq 0$, тогда $a_{3}=0$, что влечет $\omega(k)=k^{2}+a_{4} k^{4}+a_{5} k^{5}+\cdots$, и при разложении обыкновенного дифференциального уравнения в окрестности $k_{3}=0$ в ведущем нетривиальном порядке разложения получим обыкновенное дифференциальное уравнение третьего порядка

$$
-5 \omega^{\prime}\left(k_{4}\right)^{2}+8 k_{4} \omega^{\prime \prime}\left(k_{4}\right) \omega^{\prime}\left(k_{4}\right)+\omega^{\prime}\left(k_{4}\right) k_{4}^{2} \omega^{\prime \prime \prime}\left(k_{4}\right)-3 k_{4}^{2} \omega^{\prime \prime}\left(k_{4}\right)^{2}=0,
$$

единственное решение которого вида $\omega(k)=k^{2}+a_{4} k^{4}+a_{5} k^{5}+\cdots$ есть $\omega(k)=k^{2}$.

СлУчАЙ 2. Пусть $a_{3} \neq 0$, тогда $a_{2}=0$. Без потери общности можно считать, что $f(k)=k^{3}+a_{4} k^{4}+a_{5} k^{5}+\cdots$. Снова разлагая обыкновенное дифференциальное уравнение в окрестности точки $k_{3}=0$, в ведущем нетривиальном порядке получим следующее обыкновенное дифференциальное уравнение четвертого порядка:

$$
\begin{aligned}
-4 \omega^{\prime}\left(k_{4}\right)^{2} \omega^{\prime \prime \prime}\left(k_{4}\right) & +12 \omega^{\prime}\left(k_{4}\right)^{2}-k_{4} \omega^{\prime}\left(k_{4}\right)^{2} \omega^{\prime \prime \prime \prime}\left(k_{4}\right)-2 \omega^{\prime}\left(k_{4}\right) k_{4}^{2} \omega^{\prime \prime \prime}\left(k_{4}\right)+ \\
& +4 \omega^{\prime}\left(k_{4}\right) k_{4} \omega^{\prime \prime}\left(k_{4}\right) \omega^{\prime \prime \prime}\left(k_{4}\right)-18 k_{4} \omega^{\prime \prime}\left(k_{4}\right) \omega^{\prime}\left(k_{4}\right)+ \\
& +6 \omega^{\prime}\left(k_{4}\right) \omega^{\prime \prime}\left(k_{4}\right)^{2}-3 \omega^{\prime \prime}\left(k_{4}\right)^{3} k_{4}+6 k_{4}^{2} \omega^{\prime \prime}\left(k_{4}\right)^{2}=0 .
\end{aligned}
$$

Любое его решение вида $\omega(k)=k^{3}+a_{4} k^{4}+a_{5} k^{5}+\cdots$ эквивалентно $\omega(k)=k^{3}$ или $\omega(k)=k^{2} \operatorname{cth} k$ (или $\omega(k)=-k^{2} \operatorname{ctg} k$ для комплексных постоянных $a$ и $b$ в (20)). Заметим, что в случае $a_{2}=a_{3}=0$ нетривиальных решений не существует.

Полученные результаты указывают на то, что пятиволновая матрица рассеяния для уравнения (2) обращается в нуль также в случае следующего дисперсионного соотношения:

$$
\omega(k)=a k^{2} \operatorname{ctg} b k-c k,
$$

где $a, b$ и $c$ - постоянные величины. Полученное уравнение вряд ли обладает физическим смыслом, так как $\omega(k)=\infty$ при $b k=\pi n$. Однако в пределе $b \rightarrow 0, a=3 / b$ и $c=3 / b^{2}$ дисперсионное соотношение принимает вид $\omega(k)=k^{3}$, т. е. совпадает с дисперсионным соотношением для модели КдФ. Поэтому существует еще одно уравнение, которое, возможно, представляет больший интерес. Пусть $u=u(x, y, t)$ представляет собой функцию двух пространственных координат $(x, y)$, тогда $\omega=\omega(k, p)$ задается выражением

$$
\omega(k, p)=a k^{2} \operatorname{cth} a p .
$$


Матрица пятиволновой амплитуды рассеяния в этом случае обращается в нуль. В разложении Тейлора при малых $а$ получаем следующее уравнение:

$$
u_{t}+u u_{x}+\partial_{x}^{2} \partial_{y}^{-1} u=0 .
$$

То же самое уравнение можно получить, если начинать с соотношения $\omega(k, p)=$ $a k^{2} \operatorname{ctg} a p$ и произвести разложение Тейлора при малых $a$. Это уравнение напоминает, но не эквивалентно уравнению Хохлова-Заболоцкой (см., например, [10])

$$
u_{t}+u u_{x}+\partial_{y}^{2} \partial_{x}^{-1} u=0,
$$

описывающему распространение ограниченного луча в слабо нелинейной среде без дисперсии и поглощения (см. [11]).

Благодарности. Авторы благодарят др. Гиулинико за ценные обсуждения. Работа частично поддержана NSF (грант № NSF40CE-1130450) и Правительством Российской Федерации (контракт № 11.934.31.0035, подписан 25 ноября 2010 г.). Работа M. Онорато была поддержана ONR (грант № 214 N000141010991) и MIUR PRIN (грант 2012BFNWZ2).

\section{Список литературы}

[1] M. J. Ablowitz, H. Segur, Solitons, Nonlinear Evolution Equations and Inverse Scattering, London Mathematical Society Lecture Note Series, 149, Cambridge Univ. Press, Cambridge, 1991.

[2] V.E. Zakharov (ed.), What is Integrability?, Springer, Berlin, 1991.

[3] V.E. Zakharov, E. I. Schulman, Physica D, 29:3 (1988), 283-320.

[4] V.E. Zakharov, E. I. Schulman, Physica D, 1:2 (1980), 191-202.

[5] V.E. Zakharov, A. Balk, E. I. Schulman, "Conservation and scattering in nonlinear wave systems", Important Developments in Soliton Theory, eds. A. S. Fokas, V.E. Zakharov, Springer, New York, 1993, 375-404.

[6] A. I. Dyachenko, D. I. Kachulin, V. E. Zakharov, Писъма в ЖЭТФ, 98:1 (2013), 48-52.

[7] A. N. Hone, V.S. Novikov, J. Phys. A, 37:32 (2004), L399-L406.

[8] A. V. Mikhailov, V.S. Novikov, J. Phys. A, 35:22 (2002), 4775-4790, arXiv: nlin/0203055.

[9] A. I. Dyachenko, Y. V. Lvov, V.E. Zakharov, Physica D, 87:1-4 (1995), 233-261.

[10] V.E. Zakharov, "Integrable systems in multidimensional spaces", Mathematical Problems in Theoretical Physics (Berlin, August 11-20, 1981), Lecture Notes in Physics, 153, eds. R. Schrader, R. Seiler, D. A. Uhlenbrock, Springer, Berlin, 1982, 190-216.

[11] Е. А. Заболотская, Р. В. Хохлов, Акустический журнал, 15:1 (1969), 40-47. 